\title{
Statistics of Risk Aversion
}

\author{
Enzo Giacomini* \\ Wolfgang Härdle*
}

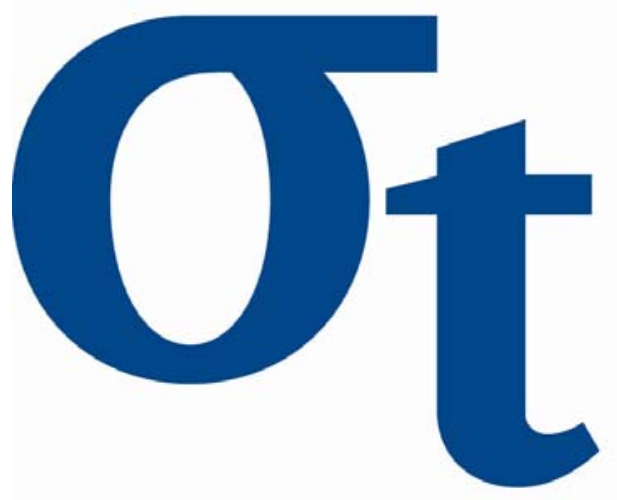

* Humboldt-Universität zu Berlin, Germany

This research was supported by the Deutsche Forschungsgemeinschaft through the SFB 649 "Economic Risk". 


\section{Statistics of Risk Aversion}

Giacomini, Enzo

Humboldt-Universität zu Berlin, CASE - Center for Applied Statistics and Economics

Spandauer Strasse 1

10178 Berlin, Germany

E-mail: giacomini@wiwi.hu-berlin.de

Härdle, Wolfgang

Humboldt-Universität zu Berlin, CASE - Center for Applied Statistics and Economics

Spandauer Strasse 1

10178 Berlin, Germany

E-mail: haerdle@wiwi.hu-berlin.de

JEL classification: C $14, \mathrm{G} 13$

Keywords: dynamic semiparametric estimation, pricing kernel, risk aversion

\section{Introduction}

Option prices are a valuable source of information concerning risk assessments from investors about future financial payoffs. The information is summarized in the state price densities (SPD), the continuous counterpart (normalized by a constant) from Arrow-Debreu security prices. Under no arbitrage assumptions the state price densities - corresponding to a risk neutral measure $Q$ - are derived from option prices as in Breeden and Litzenberger (1978). In contrast to the state price density, the historical density $p(x)$ describes the random variations of the underlying price.

In a utility based framework, investors facing financial risk have preference-indifference relations represented by a utility function. Standard economic theory enforces that the utility function is concave or equivalently, that investors are risk averse. Equilibrium and non-arbitrage arguments, as in Merton (1973), show that preferences from investors are related to the state price and historical densities, allowing to conclude the functional form of one given the functional form of the remaining two. Part of this relation is given by the pricing kernel (PK).

In this paper we investigate pricing kernels from DAX and ODAX data in a time varying approach and consider their term structure. In order to approximate and analyse the complex dynamic structure from pricing kernels and risk preferences across different maturities, we use dynamic semiparametric factor models (DSFMs). The obtained PKs exhibit risk proclivity in certain regions of returns and maturities. The dynamics of risk aversion and proclivity from investors preferences is investigated through sensitivity analysis with respect to the basis functions.

In the sequel pricing kernels are defined (section 2), their relation to utility functions and the DSFM estimation method are described (section 3). In the empirical part (section 4) pricing kernels are estimated from DAX and ODAX data sets and sensitivity analysis based on estimated loading coefficients and basis functions is performed.

\section{Pricing Kernels}

A flexible approach in a complete market is to assume that the price of a security follows a 
diffusion process described by

$$
\frac{d S_{t}}{S_{t}}=\mu\left(S_{t}, t\right) d t+\sigma\left(S_{t}, t\right) d B_{t}
$$

where $t \in[0, T], B_{t}$ is a standard Brownian motion under measure $P$. Defining

(2) $\quad \lambda_{t}=\frac{\mu\left(S_{t}, t\right)-r}{\sigma\left(S_{t}, t\right)}$

as the market price of risk, the risk neutral measure $Q$ is obtained by $\left.\frac{d Q}{d P}\right|_{\mathcal{F}_{t}}=\zeta_{t}$ where with (2)

$$
\zeta_{t}=\exp \left(-\int_{0}^{t} \lambda_{u} d B_{u}-\frac{1}{2} \int_{0}^{t} \lambda_{u}^{2} d u\right)
$$

The pricing kernel, or stochastic discount factor is defined for maturity $\tau=s-t, 0 \leq t \leq s \leq T$ as:

$$
M_{t, \tau}=e^{-r \tau} \frac{\zeta_{s}}{\zeta_{t}}
$$

Assuming the existence of a representative investor with utility function $u$, constant interest rates $r$ and defining a wealth $\left\{W_{s}\right\}$ and a consumption process $\left\{C_{s}\right\}, C_{s}=0$, the investor adjusts the amounts $\left\{\xi_{s}\right\}$ invested in the asset $S_{s}$ at times $s$ and consumes all his wealth at $T, C_{T}=W_{T}$. That means, he chooses $\xi_{s}$ via the Merton optimization problem:

$$
\max _{\left\{\xi_{s}, t \leq s \leq T\right\}} E\left[u\left(W_{T}\right)\right]
$$

subjected to $W_{s} \geq 0$ and $d W_{s}=\left\{r W_{s}+\xi_{s}(\mu-r)\right\} d s+\xi_{s} \sigma d B_{s}$. In equilibrium all wealth is invested in the asset, at the end all wealth is consumed $C_{T}=W_{T}=S_{T}$. In the Merton model the PK is path independent and (3) is equal to the marginal rate of substitution:

$$
\frac{u^{\prime}\left(S_{T}\right)}{u^{\prime}\left(S_{t}\right)}=M_{t, \tau}=e^{-r \tau} \frac{q_{t}\left(S_{T}\right)}{p_{t}\left(S_{T}\right)}
$$

Here $q_{t}, p_{t}$ denote the risk neutral and historical density at time $t$. The functional relation between the utility $u$ and the densities $q_{t}, p_{t}$ is given by

$$
u\left(S_{T}\right)=e^{-r \tau} u^{\prime}\left(S_{t}\right) \int \frac{q_{t}\left(S_{T}\right)}{p_{t}\left(S_{T}\right)} d S_{T}
$$

\section{PK Estimation with Dynamic Semiparametric Factor Models}

Breeden and Litzenberger (1978) showed how the SPD $q_{t}\left(S_{T}\right)$ may be obtained from option prices. Ait-Sahalia and Lo (1998) used the estimate:

$$
\widehat{q}_{t}\left(S_{T}\right)=\left.e^{r \tau} \frac{\partial^{2} C_{t, B S}\left\{S_{t}, K, \tau, r_{t}, \widehat{\sigma}_{t}(\kappa, \tau)\right\}}{\partial K^{2}}\right|_{K=S_{T}}
$$

where $C_{t, B S}$ is the Black-Scholes price at time $t$ and $\widehat{\sigma}_{t}(\kappa, \tau)$ is a nonparametric estimator for the implied volatility.

Implied volatilities may be estimated from option prices. On each day $i=1, \ldots, I$ there are $J_{i}$ options traded. Each intra-day trade $j=1, \ldots, J_{i}$ corresponds to an implied volatility $\sigma_{i, j}$, and a 
IVS Ticks 20000502

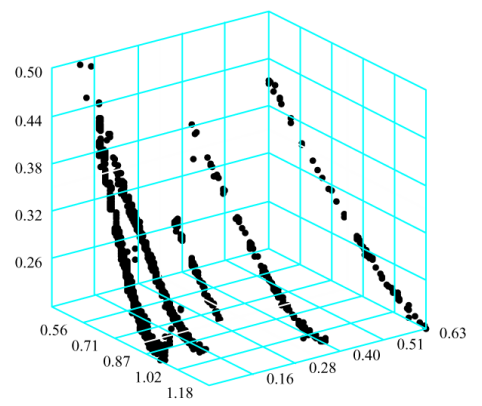

Data Design

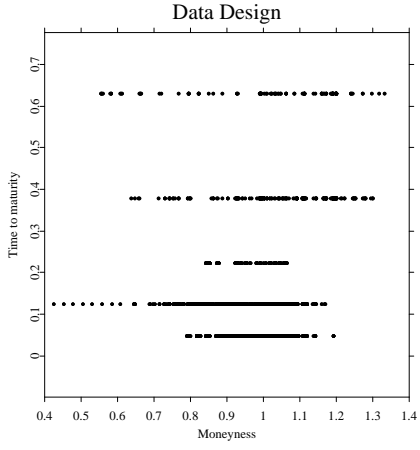

Figure 1: Call and put implied volatilities observed (left), data design (right), ODAX on 20000502

pair of moneyness and maturity $X_{i, j}=\left(\kappa_{i, j}, \tau_{i, j}\right)^{\top}$. Moneyness is defined as $\kappa_{i, j}=\frac{K}{F\left(t_{i, j}\right)}$ where $K$ is strike and $F\left(t_{i, j}\right)=S_{t_{i, j}} \exp \left(r_{\tau_{i, j}} \tau_{i, j}\right)$ are futures prices. Figure 1 depicts the implied volatilities corresponding to trades on ODAX in day 20000502 (dates are written as year, month, day).

Dynamic semi-parametric factor models (DSFM), Fengler et al. (2007), employ the time series structure of implied volatilities regressing $\log$ implied volatilities $Y_{i, j}=\log \sigma_{i, j}$ on $X_{i, j}$ using smooth basis functions $m_{l}, l=0, \ldots, L$ weighted with factor loadings $z_{i, l}$ :

$$
Y_{i, j}=\sum_{l=0}^{L} z_{i, l} m_{l}\left(X_{i, j}\right)+\varepsilon_{i, j}
$$

where $\varepsilon_{i, j}$ is noise and $z_{i, 0} \equiv 1$. Following Borak et al. (2007), the basis functions are expanded using a series estimator for functions $\psi_{k}: \mathbb{R} \rightarrow \mathbb{R}$ and coefficients $\gamma_{l, k} \in \mathbb{R}$

$$
m_{l}\left(X_{i, j}\right)=\sum_{k=1}^{K} \gamma_{l, k} \psi_{k}\left(X_{i, j}\right)
$$

Defining the matrices $Z=\left(z_{i, l}\right), \Gamma=\left(\gamma_{l, k}\right)$ for $i=1, \ldots, I, k=1, \ldots, K$ and $l=0, \ldots, L$ we obtain the least square estimators as

$$
(\widehat{\Gamma}, \widehat{Z})=\arg \min _{\Gamma \in \mathcal{G}, Z \in \mathcal{Z}} \sum_{i=1}^{I} \sum_{j=1}^{J}\left\{Y_{i, j}-z_{i}^{\top} \Gamma \psi\left(X_{i, j}\right)\right\}^{2}
$$

where $z_{i}=\left(z_{i, 0}, \ldots, z_{i, L}\right)^{\top}, \psi(x)=\left\{\psi_{1}(x), \ldots, \psi_{K}(x)\right\}^{\top}, \mathcal{G}=\mathcal{M}(L+1, K), \mathcal{Z}=\{Z \in \mathcal{M}(I, L+1)$ : $\left.z_{i, 0} \equiv 1\right\}$ and $\mathcal{M}(a, b)$ is the set of all $(a \times b)$ matrices. The estimators for the basis functions in $(7)$ are $\widehat{m}_{l}(x)=\widehat{\gamma}_{l}^{\top} \psi(x)$ where $\gamma_{l}=\left(z_{l, 1}, \ldots, z_{l, K}\right)^{\top}$. Denoting $\widehat{m}=\left(\widehat{m}_{0}, \ldots, \widehat{m}_{L}\right)^{\top}$, the implied volatility at time $i$ is estimated as $\widehat{\sigma}_{i}(\kappa, \tau)=\exp \left\{\widehat{z}_{i}^{\top} \widehat{m}(\kappa, \tau)\right\}$.

Using (6), the state price density may be approximated by

$$
\widehat{q}_{t}\left(\kappa, \tau, \widehat{z}_{t}, \widehat{m}\right)=\left.\varphi\left(d_{2}\right)\left\{\frac{1}{K \widehat{\sigma}_{t} \sqrt{\tau}}+\frac{2 d_{1}}{\widehat{\sigma}_{t}} \frac{\partial \widehat{\sigma}_{t}}{\partial K}+\frac{K \sqrt{\tau} d_{1} d_{2}}{\widehat{\sigma}_{t}}\left(\frac{\partial \widehat{\sigma}_{t}}{\partial K}\right)^{2}+K \sqrt{\tau} \frac{\partial^{2} \widehat{\sigma}_{t}}{\partial K^{2}}\right\}\right|_{K=S_{T}}
$$

where $\varphi(x)$ is the standard normal pdf, $d_{1}=\frac{\log \left(\frac{S_{t}}{K}\right)+\left(r+\frac{1}{2} \widehat{\sigma}_{t}^{2}\right) \tau}{\widehat{\sigma}_{t} \sqrt{\tau}}$ and $d_{2}=d_{1}-\widehat{\sigma}_{t} \sqrt{\tau}$. As in Ait-Sahalia and Lo $(2000)$ we define an estimate $\widehat{M}_{t}(\kappa, \tau)$ of the PK as the ratio between the estimated SPD and 


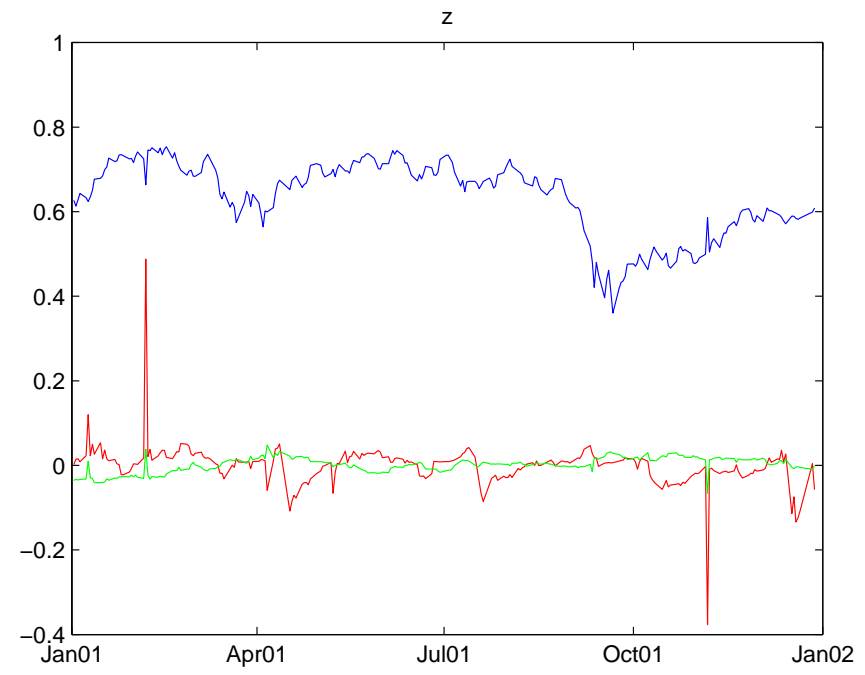

Figure 2: Loading factors $\widehat{z}_{t, l}, l=1,2,3$ from the top

the estimated $p$ :

$$
\widehat{M}_{t}\left(\kappa, \tau, \widehat{z}_{t}, \widehat{m}\right)=e^{-r_{t} \tau} \frac{\widehat{q}_{t}\left(\kappa, \tau, \widehat{z_{t}}, \widehat{m}\right)}{\widehat{p}_{t}(\kappa, \tau)}
$$

Here $\widehat{p}_{t}$ is estimated by a $\operatorname{GARCH}(1,1)$ model. It is our interest in this paper to examine the dynamic structure of (9).

\section{Empirical Results}

Here SPDs and PKs are estimated from intraday DAX and ODAX data. The options data ranges from 20010101 to 20020101 corresponding to 253 trading days. The SPD $\hat{q}_{t}$ (8) is estimated with 4 basis functions. The historical density $\hat{p}_{t}$ is estimated with $\operatorname{GARCH}(1,1)$ from the last 240 observations. The time series of loading factors $\left\{\widehat{z}_{t, l}\right\}$ and basis functions $\widehat{m}_{l}, l=0, \ldots, 3, t=1, \ldots, 253$ are in figures 2 and 3. The summary statistics from loading factors series are in table 1. From (8) and (9) we obtain a sequence of 253 SPDs and PKs moving on time over a grid of moneyness and maturities, figure 4 shows one shot of this sequence, at day 20010710.

Risk averse utilities $u$ are concave. Hence, (4) implies that under risk aversion pricing kernels are monotone decreasing in moneyness. Figure 4 shows that DAX PKs - similarly to S\&P PKs, Ait-Sahalia and Lo (2000) - present risk proclivity at certain moneyness levels (PKs are increasing between 0.9 and 1 ) and these levels vary across maturities, hence we verify the empirical pricing kernel paradox.

In order to analyse the dynamics of risk aversion and proclivity implied in PKs we focus on the SPDs, as these contain information about preferences from investors. In figure 5 we can see that the mean of $\widehat{q}_{t}\left(S_{T}\right)$ and the factor loadings $\widehat{z}_{t, 1}$ from the basis function $\widehat{m}_{1}$ are correlated. We investigate the influence of the basis functions $\widehat{m}_{l}$ on the mean, variance and skewness of SPDs by sensitivity analysis to changes in the loadings $\left\{\widehat{z}_{t, l}\right\}$ : the SPDs are recalculated with new loadings, where one of the coefficients varies linearly and the remaining are hold fixed.

We define scenario loadings $W^{l}$ corresponding to a linear increase on factor $l$ in $N$ steps from 
Table 1: Loading factors, descriptive statistics

\begin{tabular}{c|ccccc}
\hline \hline & min & max & median & mean & std.dev. \\
\hline$\widehat{z}_{t, 1}$ & 0.36 & 0.75 & 0.66 & 0.63 & 0.09 \\
$\widehat{z}_{t, 2}$ & -0.37 & 0.49 & 0.01 & 0.00 & 0.05 \\
$\widehat{z}_{t, 3}$ & -0.07 & 0.05 & 0.00 & 0.00 & 0.02 \\
\hline \hline
\end{tabular}
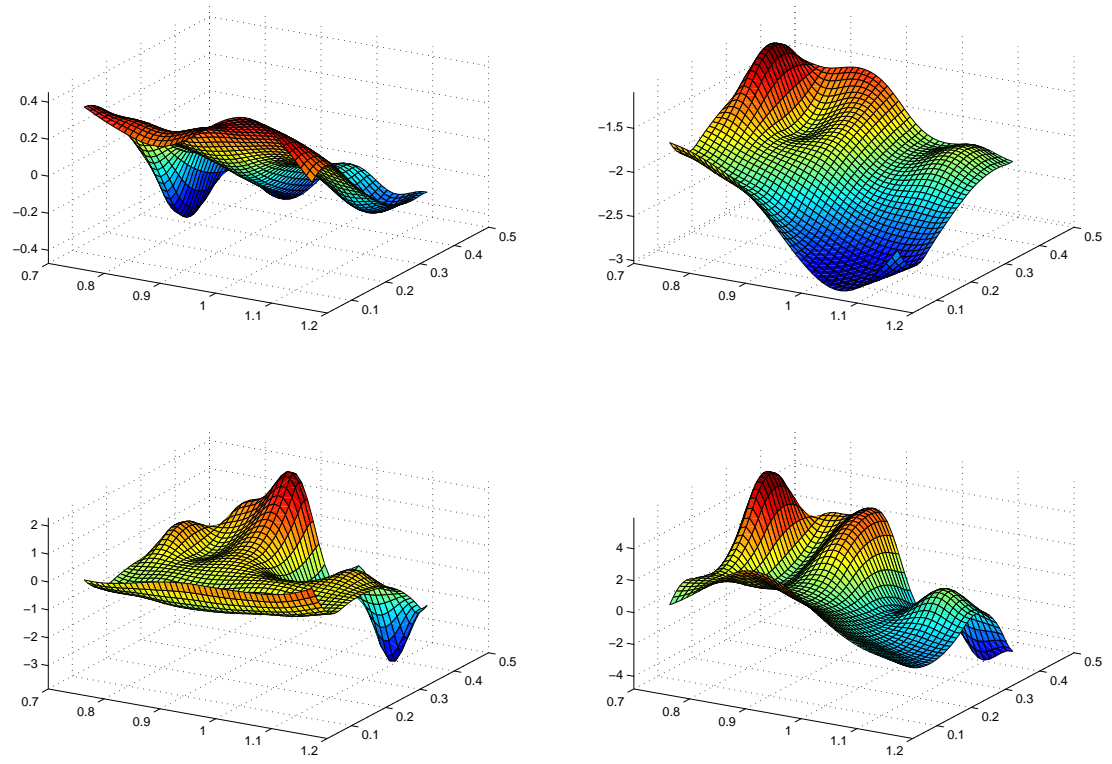

Figure 3: Basis functions $\widehat{m}_{l}, l=0, \ldots, 3$ clockwise
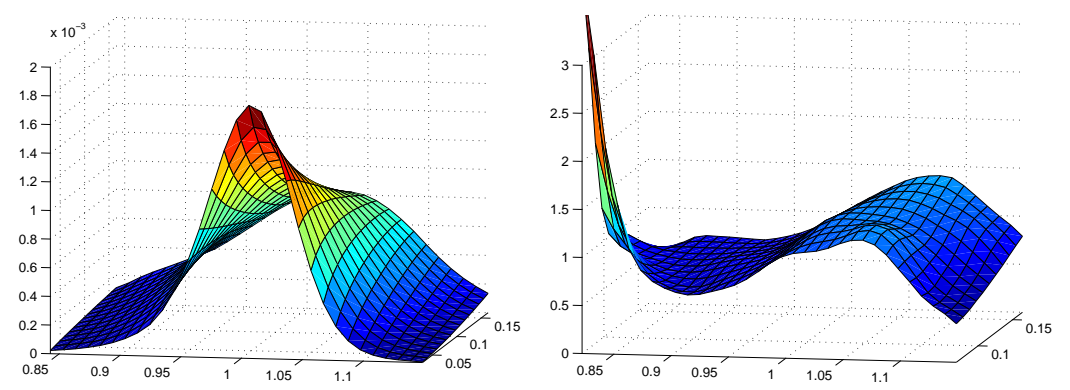

Figure 4: Estimated SPD (left) and PK (right) across $\kappa$ and $\tau$ at $t=20010710$ 


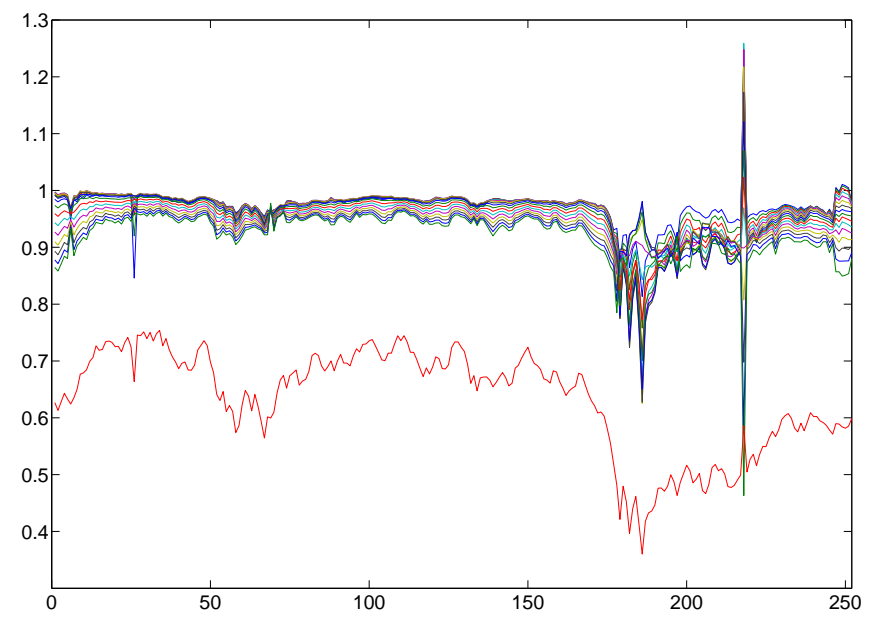

Figure 5: Mean from SPD for $\tau=25, \ldots, 75$ days (above), $\widehat{z}_{t, 1}$ (below)

levels $d_{l}$ to $u_{l}$. The remaining coefficients are held constant at median of estimated values. The matrices $W^{l}=\left(w_{n, j}^{l}\right), l, j=0, \ldots, 3$, and $n=1, \ldots, N$ contain the scenario loadings to factor $l$ as

$$
w_{n, j}^{l}=\left\{d_{j}+\frac{n-1}{N-1}\left(u_{j}-d_{j}\right)\right\} \mathbf{1}(j=l)+\operatorname{med}\left(\widehat{z}_{t, j}\right) \mathbf{1}(j \neq l)
$$

with levels given by $d_{l}=\min \widehat{z}_{t, l}-0.5\left|\min \widehat{z}_{t, l}\right|$ and $u_{l}=\max \widehat{z}_{t, l}+0.5\left|\max \widehat{z}_{t, l}\right|$. SPDs estimated with loadings $W^{l}$ are under influence of variations in factor $l$ while the remaining factor are constant at a typical level (median). The observed changes in mean, variance and skewness may be considered as a typical effect of variation in factor $l$.

Figure 6 shows the mean, variance and skewness of SPD obtained with $W^{l}$, for $l=1,2,3$, $N=50$ and three different maturities (25, 40 and 75 days) plotted against $n$. From the middle column, we verify that increasing factor 2 decreases mean while increasing variance and skewness for short maturities (full lines) and increases mean while decreasing variance and skewness for long maturities (dashed lines). From the left column, we verify that increasing factor 1 increases mean while decreasing variance and skewness for all maturities.

Figure 7 plots PKs obtained with $W^{l}$, for $l=1,2,3, N=50$ and two maturities, short (20) and long (75 days), against moneyness. The pricing kernels are calculated with weak $(n=1)$ medium $(25)$ and strong (50) loadings from each factor. The influence of factor 1 is represented by the left pair in figure 7: for both maturities weak loadings result in risk proclivity for $\kappa>1$ while strong loadings in risk proclivity for $\kappa<1$.

In the middle pair we see that factor 2 has the opposite influence at short maturities: weak loadings result in risk proclivity for $\kappa<1$, strong loadings for $\kappa>1$. This effect is not clear at long maturities. Increasing loadings of the factor 3 shifts the risk proclivity region to higher values of $\kappa$ in both maturities, as observed in the right pair of figure 7 . 

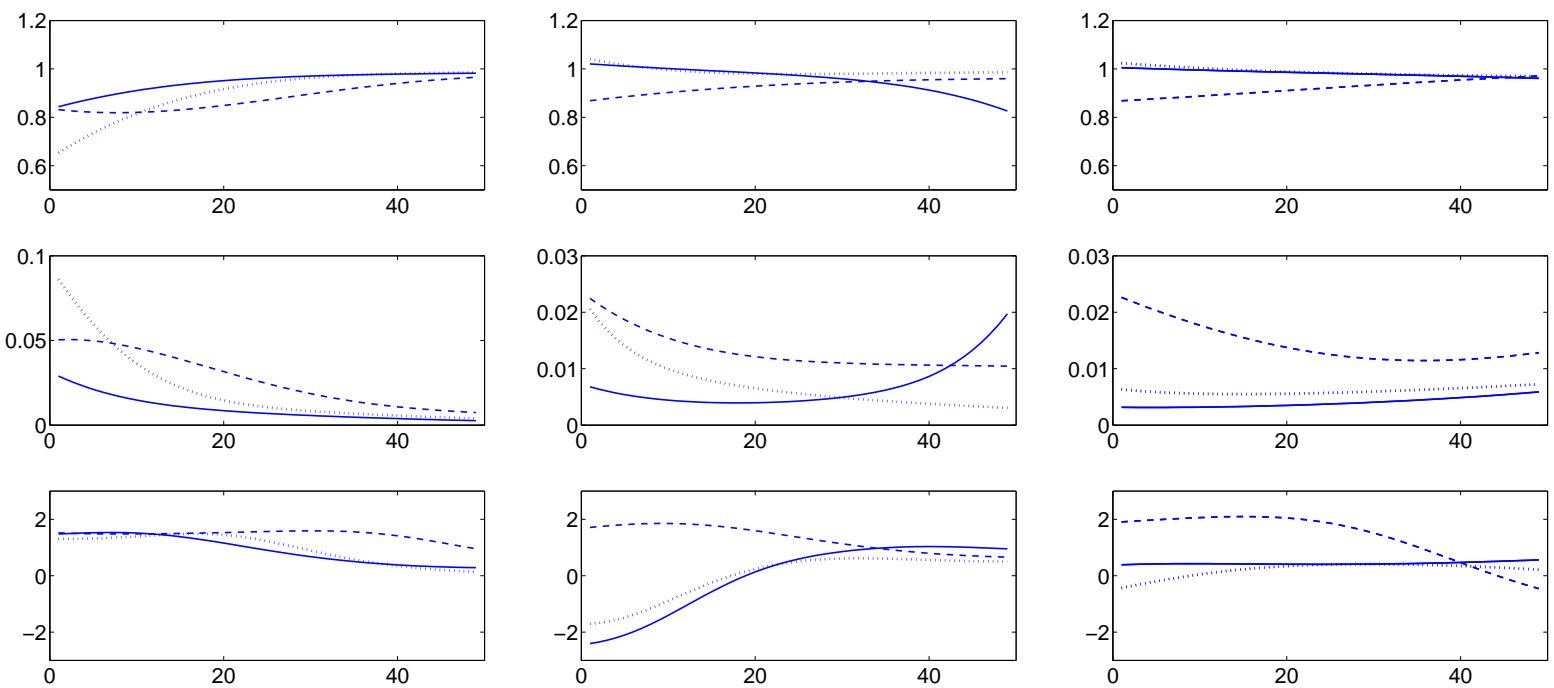

Figure 6: Mean, variance and skewness from SPD (from the top) plotted against n. For $W^{1}, W^{2}$ and $W^{3}$ (from the left), $\tau=25$ (full), 40 (dotted) and 75 (dashed) days, $N=50$
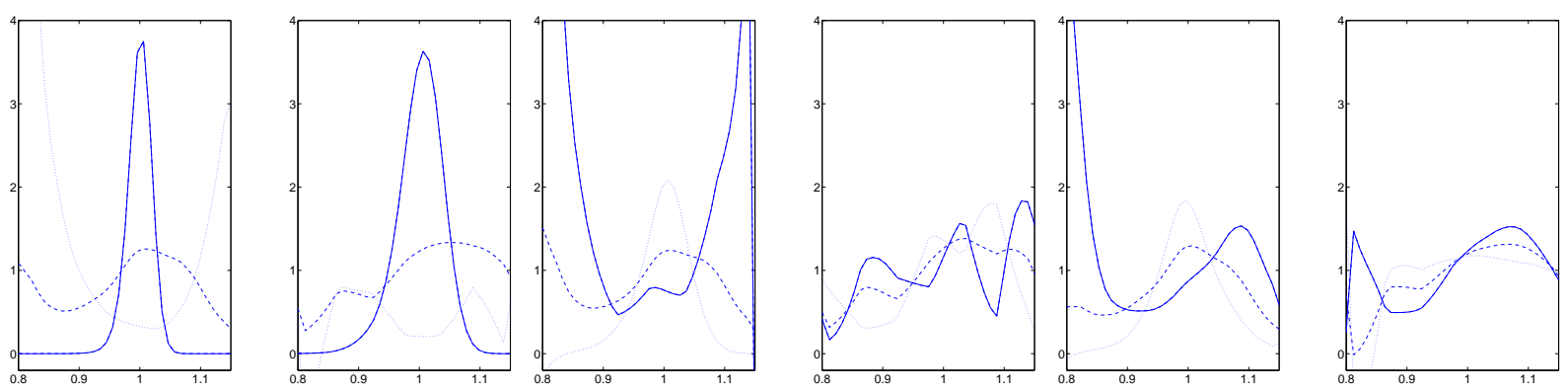

Figure 7: PK obtained with loadings $W^{1}$ (left pair), $W^{2}$ (middle pair) and $W^{3}$ (right pair), $\tau=25$ (first) and 75 (second in each pair) days, $n=1$ (dotted), 25 (dashed) and 50 (full) 


\section{REFERENCES}

Ait-Sahalia, Y. and Lo, A. (1998). Nonparametric estimation of state-price densities implicit in financial asset prices. Journal of Finance, 53:499-547.

Ait-Sahalia, Y. and Lo, A. (2000). Nonparametric risk management and implied risk aversion. Journal of Econometrics, 94:9-51.

Borak, S., Härdle, W., Mammen, E., and Park, B. (2007). Time series modelling with semiparametric factor dynamics. Discussion paper, SFB 649 - Humboldt-Universität zu Berlin, 2007-23.

Breeden, D. and Litzenberger, R. (1978). Prices of state-contingent claims implicit in options prices. Journal of Business, 51:621-651.

Debreu, G. (1959). Theory of Value. An Axiomatic Analysis of Economic Equilibrium. John Wiley and Sons, Inc., New York.

Fengler, M., Härdle, W., and Mammen, E. (2007). A semiparametric factor model for implied volatility surface dynamics. Journal of Financial Econometrics, 5:189-218.

Merton, R. (1973). Theory of rational option pricing. The Bell Journal of Economics and Management Science, $4: 141-183$

\section{ABSTRACT}

Information about risk preferences from investors is essential for modelling a wide range of quantitative finance applications. Valuable information related to preferences can be extracted from option prices through pricing kernels. In this paper, pricing kernels and their term structure are estimated in a time varying approach from DAX and ODAX data using dynamic semiparametric factor model (DSFM). DSFM smooths in time and space simultaneously, approximating complex dynamic structures by basis functions and a time series of loading coefficients. Contradicting standard risk aversion assumptions, the estimated pricing kernels indicate risk proclivity in certain levels of return. The analysis of the time series of loading coefficients allows a better understanding of the dynamic behaviour from investors preferences towards risk.

\section{AKNOWLEDGEMENTS}

The Financial support from the Deutsche Forschungsgemeinschaft via SFB 649 "Ökonomisches Risiko", Humboldt-Universität zu Berlin is gratefully acknowledged. 


\section{SFB 649 Discussion Paper Series 2007}

For a complete list of Discussion Papers published by the SFB 649, please visit http://sfb649.wiwi.hu-berlin.de.

001 "Trade Liberalisation, Process and Product Innovation, and Relative Skill Demand" by Sebastian Braun, January 2007.

002 "Robust Risk Management. Accounting for Nonstationarity and Heavy Tails" by Ying Chen and Vladimir Spokoiny, January 2007.

003 "Explaining Asset Prices with External Habits and Wage Rigidities in a DSGE Model." by Harald Uhlig, January 2007.

004 "Volatility and Causality in Asia Pacific Financial Markets" by Enzo Weber, January 2007.

005 "Quantile Sieve Estimates For Time Series" by Jürgen Franke, JeanPierre Stockis and Joseph Tadjuidje, February 2007.

006 "Real Origins of the Great Depression: Monopolistic Competition, Union Power, and the American Business Cycle in the 1920s" by Monique Ebell and Albrecht Ritschl, February 2007.

007 "Rules, Discretion or Reputation? Monetary Policies and the Efficiency of Financial Markets in Germany, 14th to 16th Centuries" by Oliver Volckart, February 2007.

008 "Sectoral Transformation, Turbulence, and Labour Market Dynamics in Germany" by Ronald Bachmann and Michael C. Burda, February 2007.

009 "Union Wage Compression in a Right-to-Manage Model" by Thorsten Vogel, February 2007.

010 "On $\sigma$-additive robust representation of convex risk measures for unbounded financial positions in the presence of uncertainty about the market model" by Volker Krätschmer, March 2007.

011 "Media Coverage and Macroeconomic Information Processing" by Alexandra Niessen, March 2007.

012 "Are Correlations Constant Over Time? Application of the CC-TRIG to Return Series from Different Asset Classes." by Matthias Fischer, March 2007.

013 "Uncertain Paternity, Mating Market Failure, and the Institution of Marriage" by Dirk Bethmann and Michael Kvasnicka, March 2007.

014 "What Happened to the Transatlantic Capital Market Relations?" by Enzo Weber, March 2007.

015 "Who Leads Financial Markets?" by Enzo Weber, April 2007.

016 "Fiscal Policy Rules in Practice" by Andreas Thams, April 2007.

017 "Empirical Pricing Kernels and Investor Preferences" by Kai Detlefsen, Wolfgang Härdle and Rouslan Moro, April 2007.

018 "Simultaneous Causality in International Trade" by Enzo Weber, April 2007.

019 "Regional and Outward Economic Integration in South-East Asia" by Enzo Weber, April 2007.

020 "Computational Statistics and Data Visualization" by Antony Unwin, Chun-houh Chen and Wolfgang Härdle, April 2007.

021 "Ideology Without Ideologists" by Lydia Mechtenberg, April 2007.

022 "A Generalized ARFIMA Process with Markov-Switching Fractional Differencing Parameter" by Wen-Jen Tsay and Wolfgang Härdle, April 2007.

\section{SFB 649, Spandauer Straße 1, D-10178 Berlin} http:/ / sfb649.wiwi.hu-berlin.de 
023 "Time Series Modelling with Semiparametric Factor Dynamics" by Szymon Borak, Wolfgang Härdle, Enno Mammen and Byeong U. Park, April 2007.

024 "From Animal Baits to Investors' Preference: Estimating and Demixing of the Weight Function in Semiparametric Models for Biased Samples" by Ya'acov Ritov and Wolfgang Härdle, May 2007.

025 "Statistics of Risk Aversion" by Enzo Giacomini and Wolfgang Härdle, May 2007. 